\title{
Precise crystal regulation and harvest by constructing confined flexible droplet crystallizer on 3D-printed sessile platform
}

\section{Zhijie Yuan}

Dalian University of Technology

Xiaobin Jiang ( $\nabla$ xbjiang@dlut.edu.cn )

Dalian University of Technology https://orcid.org/0000-0003-0262-4354

\section{Yuchao Niu}

Dalian University of Technology

\section{Mengyuan Wu}

Dalian University of Technology

\section{Yingshuang Meng}

Dalian University of Technology

\section{Zhengtao Li}

Dalian University of Technology

\section{Xuehua Ruan}

Dalian University of Technology

\section{Xiangcun Li}

Dalian University of Technology

\section{Wu Xiao}

Dalian University of Technology

\section{Xuemei Wu}

Dalian University of Technology

\section{Gaohong He}

Dalian University of Technology

\section{Article}

Keywords: 3D printed sessile platform, confined flexile droplet crystallizer, synergized micro-flows, capillary flow, Marangoni flow, crystal regulation

Posted Date: June 1st, 2021

DOI: https://doi.org/10.21203/rs.3.rs-515125/v1 
License: (c) (i) This work is licensed under a Creative Commons Attribution 4.0 International License. Read Full License 


\section{Abstract}

Precise regulation of the mass-heat-momentum transfer carried great implications to the crystallization behavior in microscale droplet, which is one of the core concerns in crystal engineering. Herein, a confined flexible droplet crystallizer (CFDC) was facilely constructed on designed 3D-printed, matrix-type regular sessile platforms. Owing to the flexible morphology of CFDC, the synergized micro-flows generated by the integration of capillary flow and Marangoni flow in CFDC could be precisely controlled, which was validated from both experimental and simulative aspects. Standard cubic crystal was manufactured at the sessile platform center after evaporating an optimized CFDC (with $120^{\circ}$ featured angle), where the synergized micro-flows created the perfect flow zone to balance the mass transfer and particle location. In addition, larger standard cubic crystal could be obtained via simply scale-up the platform (16 times) or supplementary feeding (secondary to forth feeding), manifesting great potential for high-quality crystal parallel preparation and harvest.

\section{Introduction}

Droplet based crystallization attracted remarkable attentions owing to the superiorities of crystal screening, particle harvest and theoretical research with limited solution volume and flexible solution condition, such as nano material fabrication, DNA microarray design, and protein crystallization, etc ${ }^{1-10}$. Generally, the regulation on droplet crystallization was focused on the modulation of antisolvent, heterogeneous interface, and evaporative atmosphere (temperature and wettability, etc.) ${ }^{11-19}$. The patterned heterogeneous interface was designed to control the nucleation location of insulin and lysozyme crystals, facilitating the crystal harvest ${ }^{20}$. The strategy exhibited a preliminary regulation on the biomacromolecule crystals morphology. While, the undergoing mechanism relevant to crystallization and particle transfer during the droplet crystallization was the perpetual concern.

Micro-flows in the droplet were originated by multiple impactors varied from solvent compensation, particle sedimentation, solution density and surface tension gradient, which had significant impact on the mass, heat, and momentum transfer inside droplets ${ }^{21-26}$. Fundamental physical phenomena (such as convection, wetting, pinning and migration of contact line, etc.) were all affected by micro-flows $2,27,28$. In principle, micro-flows inside droplet were deemed to be an integration of capillary flow and Marangoni flow $27,29,30$. The capillary flow was commonly induced by the asymmetrical evaporation on the gas-liquid interface of droplet and the Marangoni flow was driven by the surface tension gradient evoked by temperature and concentration gradients ${ }^{31-37}$. The uncontrollable micro-flows inside droplet caused unpredictable feed status and crystal morphology ${ }^{36}$.

Numerous discussions had been developed to evaluate the micro-flows mechanism during droplet crystallization ${ }^{38-42}$. Owing to the weak Marangoni effect, micro-flows inside droplet were dominated by radially outward capillary flow towards contact line, which was first observed by Deegan and the corresponding particle deposition was named as 'coffee-ring'37,43. This phenomenon resulted in the 
contact line migration of solutes, thereby the crystal nucleation, growth, and the final morphology would be impacted by the unstable gas-liquid-solid interface, which became a major barrier on the conveyance and harvest of high-quality crystal ${ }^{40}$. Thus, researchers attempted to regulate the micro-flows by enhancing the Marangoni flow intensity, constructing a perfect flow zone for crystal growth and transfer, further obtaining an ideal crystal. Soluble surfactants were added into the crystallization solution to enhance the Marangoni flow intensity inside the droplet. And then a localized vortex was formed at the contact line region, such radially inward vortex prevented the particles from reaching the contact line, leading to a formation of uniform deposition rather than a common coffee-ring ${ }^{44-46}$. Afterwards, the further intensified Marangoni flow was realized by modifying the crystallization platform surface, which enhanced the localized vortex and reversed the migration of particles. Thereby, the particles were conveyed towards droplet center, resulting in a construct of dome deposition rather than the coffee-ring and uniform deposition ${ }^{26,27}$.Thus, an ideal synergy of capillary flow and Marangoni flow might achieve the accurate regulation of deposition and the preparation of diverse crystals. However, these regulations of micro-flows replied on the modification of heterogeneous interface properties and effective flow space, which was severely restricted the clear revelation of micro-flows mechanism and the universal harvest of high purity crystal in proper crystallization circumstances. One great challenge was how to construct an ideal droplet crystallizer to accurately control the synergy of capillary flow and Marangoni flow without additional impactors.

Herein, we achieved the precisely fabrication of hydrophilic sessile platform by three-dimensional (3D) printing technology, whose hydrophilicity endowed the platform with a function of sucking the droplet. In this way, the sessile platform can break through its limitation of inherent property (i.e., hydrophilicity) to restrain the spread and shrinkage of droplet on sessile platform while the droplet volume was regulated, realizing the flexible and controlled gas-liquid interface. Thereby, the droplets with different morphologies (i.e., contact angle, aspect ratio) could be effectively constructed and confined on sessile platform due to the surface tension and affinity between flexible droplet and hydrophilic platform, meaning an achievement of confined flexible droplet crystallizer (CFDC). Benefiting the on-line monitoring camera and computational fluid dynamics simulation (CFD), $\mathrm{NaCl}$ aqueous solution was utilized to systematically investigate the droplet crystallization process and further reveal the subtly synergy between capillary and Marangoni flow. In this case, an ideal cubic crystal was obtained at the sessile platform center with high successful rate, which was also scaled up (about 16 times) on the cubic crystal size. This developed CFDC rectified the crystallization process with feasible operation on the micro and matrix-type platform, opening an avenue to the high-quality crystal preparation, scale up design, precise crystal screen and harvest for the micro droplet crystallization.

\section{Results}

3D printed sessile platform and construction of confined flexible droplet crystallizer (CFDC). In this study, the CFDC with different volume and morphology were subtly created on the self-designed 3D printed sessile platform. Hydrophilic polyacrylic acid (PAA) was selected as main material to precisely 
manufacture the sessile platform (radius of $600 \mu \mathrm{m}$ and height of $800 \mu \mathrm{m}$ ) by 3D printing as exhibited in Supplementary Fig. 1, which can effectively restrain the spread and shrinkage of droplet. Thus, a series of CFDCs were facilely constructed on sessile platform by tuning the droplet volume from $0.1 \mu \mathrm{L}, 0.2 \mu \mathrm{L}$, to $2.0 \mu \mathrm{L}$ via precise droplet dripping system (Fig. 1a-C). More detailed demonstrations on the CFDC were provided in Supplementary Fig. 2. Correspondingly, the contact angle of CFDC was increased from $51^{\circ}$, $60^{\circ}$, to $140^{\circ}$ with a stable location on the sessile platform, respectively. Notably, to avoid the electrostatic interaction between platform and solute, the sessile platform was immersed in ammonium hydroxide solution for $48 \mathrm{~h}$ to neutralize the residual carboxyl groups before the CFDC construction, which was proved by the reduced negative zeta potential from $-26.1 \mathrm{mV}$ to $-2.9 \mathrm{mV}$ (Fig. 1e). The neutralized property was also verified by the increased $\mathrm{N}$ element and reduced $\mathrm{O}$ element, which were detected by energydispersive spectroscopy (EDS) and X-ray photoelectron spectroscopy (XPS) as demonstrated in Supplementary Figs. 3, 4. Meanwhile, the hydrophilicity and flat surface of sessile platform was maintained after the neutralization treatment, which was testified by the inherent contact angle of $77^{\circ}$ and low roughness of $\mathrm{Ra}=30 \mathrm{~nm}$ in scanning electron microscopy (SEM) and atomic force microscopy (AFM) images (Fig. 1a, d). All these results manifested the fabrication of a flat, hydrophilic, and neutral sessile platform, facilitating the exploration of droplet crystallization process.

Synergized micro-flows mechanism inside CFDC. Benefiting from the implementation of various CFDCs on the sessile platform, we unfolded the synergized micro-flows mechanism inside CFDC. CFDCs with contact angle of $90^{\circ}, 120^{\circ}$, and $140^{\circ}$ were selected to explore the different synergized micro-flows mechanism induced by the shape of CFDC. Firstly, $\mathrm{CuSO}_{4}$ and $\mathrm{Na}_{2} \mathrm{SO}_{4}$ aqueous solutions were used to evaluate the crystal growth and deposition due to the virtue of dendritic crystal on illustrating the initial nucleation sites and growth pathway. The classic dome deposition was formed at the center of sessile platform when the initial contact angle of CFDC was $120^{\circ}$ as depicted in Fig. $2 \mathrm{~b}$. Unfortunately, small CFDC (contact angle $90^{\circ}$ ) endowed deposition with a classic coffee-ring deposition along the contact line while the large CFDC $\left(140^{\circ}\right)$ left a random aggregation (Fig. 2a, C). In particular, the nucleation sites of dome deposition and coffee-ring deposition were separately focused on the interior and contact line of sessile platform. And then both crystals grew towards the center of sessile platform, hinting a radially inward vortex. While, the nucleation sites and growth pathways would become random when the contact angle was $140^{\circ}$. These phenomena implied that the morphology of CFDC can effectively and simply regulate the crystal deposition morphology via the potential synergized micro-flows inside CFDC.

To explore the evolution of the deposition morphology, neutral nanoparticle $\mathrm{SiO}_{2}$, with radius of $200 \mathrm{~nm}$, was introduced to simulate the small crystal particle transfer during the crystallization process via eliminating the impact of the solution concentration gradient. In this scenario, the compensation of solution was replenished from everywhere inside droplet driven by the surface tension, rather than from the droplet center (lowest concentration region) due to the concentration difference. Under this condition, Marangoni flow can dominate the formation of synergized micro-flows by impairing the effect of capillary flow, which was clearly verified by the radially inward orientation of micro-flows in all CFDCs as shown in Supplementary Video 1. Furthermore, the trajectory of one nanoparticle was recorded via on-line 
monitoring camera to calculate the translational speed of nanoparticle, which can illustrate the Marangoni flow intensities in different CFDCs (Fig. 2d and Supplementary Fig. 5). These results demonstrated that the motion of suspended nanoparticles was accelerated with the increase of initial CFDC contact angle from $90^{\circ}$ to $140^{\circ}$, indicating the enhanced intensity of Marangoni flow. After drying the solvent, a uniform deposition of nanoparticles was observed on the sessile platform after evaporating a small CFDC $\left(90^{\circ}\right)$ as displayed in Fig. 2a. It should be attributed to the weak Marangoni flow inside the small CFDC $\left(90^{\circ}\right)$, which is incapable of transporting the particles towards sessile platform center. And then the increased initial contact angle $\left(120^{\circ}\right)$ endowed the Marangoni flow with stronger driven force for the nanoparticle transfer, realizing an aggregation of particles towards the platform center (Fig. 2b). Identical tendency was further intensified when the initial contact angle of CFDC increased from $120^{\circ}$ to $140^{\circ}$, implying much stronger Marangoni flow on conveying nanoparticles towards the center of the sessile platform. Therefore, this aggregation was further intensified, and a dome deposition was shown in Fig. 2c. These findings distinctly confirmed that the deposition morphology can be controlled by modifying CFDC morphology via the regulation of Marangoni flow intensity.

Next, to further investigate the undergoing mechanism of the synergized Marangoni flow and capillary flow in CFDC, the computational fluid dynamics (CFD) simulations were implemented to analyze the temperature, concentration, and velocity distributions inside the CFDCs ( $20 \% \mathrm{NaCl}$ aqueous solution) as depicted in Fig. 3. The corresponding contours and independence verifications were provided in Supplementary Fig. 6. Firstly, these distributions along the axes of CFDCs was supplied to exploited the synergized micro-flows mechanism in Fig. 3a-c. Noting that a corner of temperature distribution and a peak of concentration distribution along the axis were observed in Fig. 3a, b, which indicated a special region (center shaft region) with the lowest temperature and highest concentration was formed inside the CFDCs as marked in Supplementary 7. Driven by the temperature and concentration gradients, a radially inward vortex was created at the contact line region while another radially outward vortex was appeared at the dome region, which transported the solution towards the center shaft region, realizing the highest velocity at center shaft region as manifested in Fig. 3c. With the increased contact angle, a connection was constructed between the vortex at dome region and another enhanced vortex at contact line region, which inverted the transfer of solutes and or micro-crystals towards contact line. Concretely, when contact angle was increased from $90^{\circ}$ to $140^{\circ}$, the reduced corner temperature (from 296.5 to $293.7 \mathrm{~K}$ ) and the increased concentration peak value (from $21.4 \%$ to $24.3 \%$ ) declared the enhanced temperature and concentration gradients, certifying the strengthened Marangoni flow intensity. Together with the upshifts of the temperature corner and concentration peak position from 0.34 to $1.06 \mathrm{~mm}$, the radially inward vortex would be constantly enhanced and enlarged, which was evidenced by the similar variations for the peak value and position of velocity distribution. More detailed distributions for other CFDCs were validated in Supplementary Fig. 8, which were consistent with the aforementioned experimental results.

In addition, the temperature, concentration, and velocity distributions along the gas-liquid interface of CFDC were also obtained from CFD simulations to explain the synergized micro-flows. Specifically, the dome of all CFDCs possessed the lowest temperature, in which the solution was transported towards the dome region along the gas-liquid interface following the temperature gradient (Fig. $3 \mathrm{~d}$ and 
Supplementary Fig. 9). This was similar to the detection of infrared thermal imager as displayed in Supplementary Fig. 10. Combined with the concentration gradient (Supplementary Fig. 11), we found that the solution would be transported along the two vortexes at the dome and contact line regions, which was kept in agreement with the effect of the temperature and concentration gradients along the axis. Besides, the temperature and concentration gradients were also boosted with the increase of the CFDC initial contact angle. These conjectures were intuitively proved by the fully developed velocity vectors projection on the cross-section plane of CFDC in Fig. 3e. With the increase of CFDC contact angle, induced by the raised center shaft region, the vortex at the contact line region was constantly enhanced until the CFDC was thoroughly occupied $\left(140^{\circ}\right)$ by invading the vortex at dome region (Fig. 3e). Conversely, the vortex at dome region would be reduced until it was completely devoured.

In a word, the Marangoni flow intensity was constantly enhanced by increasing the CFDC contact angle, thereby synergized micro-flows were subtly regulated by controlling the integration of capillary flow and Marangoni flow. As illustrated in Fig. $3 f$, for the $30^{\circ}$ CFDC, the synergized micro-flows were dominated by the radially outward capillary flow due to the weak Marangoni flow intensity (Ma, 154.1). Thus, the solution was transported from center to contact line. Noting that the Ma was calculated by the CFD simulation as listed in Supplementary Table 1. By contrast, when the contact angle was increased to $60^{\circ}$, a small radially inward vortex at contact line region was induced by the synergy of capillary flow and intensive Marangoni flow (Ma, 452.4), realizing a solution transfer away from the contact line of CFDC. Then, the intensity (i.e., size and velocity) of such radially inward vortex was further strengthened along with the increased contact angle from $60^{\circ}$ to $140^{\circ}$ ascribed to the enhanced Marangoni flow intensity (Ma, from 452.4 to 2493.1). Notably, when it comes to $140^{\circ}$, the synergized micro-flows were dominated by the strong Marangoni flow (Ma, 2493.1), exhibiting a big radially inward vortex as illustrated in Fig. 3f, where the sharp changes from $120^{\circ}$ to $140^{\circ}$ could be exposed by the velocity vector of $130^{\circ}$ in Supplementary Fig. 12. These interesting findings hinted that a magic shape and featured angle can be obtained by regulating the morphology of CFDC, which achieved the ideal synergized micro-flows by integrating the Marangoni flow and capillary flow, presenting great potential on the precise modulation of crystal preparation. Meanwhile, the flexibility of the gas-liquid interface and the confinement of spatial structure inside CFDC opened an avenue for the regulation of complex combined flow.

Precise regulation of targeted crystal morphology. Following above guidance about the synergized microflows, $\mathrm{NaCl}$ aqueous solution was introduced to further evaluate the crystal morphology due to the insensitive solubility of $\mathrm{NaCl}$ to temperature and humidity. First of all, various nanoliter CFDCs were constructed on sessile platform with controllable contact angle (from $90^{\circ}, 120^{\circ}$ to $140^{\circ}$ ). Subsequently, the crystallization process of CFDC was captured by on-line camera and the crystal morphology was distinctly analyzed as shown in Fig. 4. Particularly, a coffee-ring crystal along the sessile platform edge was acquired after drying a small CFDC $\left(90^{\circ}\right)$ as demonstrated in Fig. $4 a$, b. This should be attributed to the capillary flow dominated micro-flows due to the synergy of capillary flow and weak Marangoni flow ( $\mathrm{Ma}, 828.1<1000)$, which conveyed the solutes and/or micro-crystals towards the contact line region and generated the coffee-ring crystal as illustrated in Fig. 4g. And then the Marangoni flow (Ma, 1373.7) was enhanced in the moderate CFDC $\left(120^{\circ}\right)$, which realized a perfectly synergized micro-flows by controlling 
the match of capillary flow and Marangoni flow. Thus, the synergized micro-flows conveyed the solutes and/or micro-crystals towards the center shaft region, and ultimately a standard cubic crystal was assembled at the center of the sessile platform as displayed in Fig. 4c, d. Unfortunately, for the large CFDC $\left(140^{\circ}\right)$, the match of capillary flow and Marangoni flow was destroyed by the excessive Marangoni flow intensity (Ma, 2493.1>2000). The synergized micro-flows were dominated by the Marangoni flow, where the overhigh solutes and/or micro-crystals transfer velocity caused drastic collision between microcrystals and sessile platform, resulting in a formation of random crystal as shown in Fig 4e-g. These phenomena confirmed that the shape of CFDC possessed pronounced impact on the modulation of the synergized micro-flows by regulating the Marangoni flow intensity, further controlling the crystal morphology and deposition.

By virtue of this perfect synergized micro-flows inside moderate CFDC $\left(120^{\circ}\right)$, we furtherly exploited the massive production of such cubic crystal in spatial (scale-up, enlarged platform size) and temporal (stage-up, supplementary feeding droplet) detail by expanding the CFDC size and replenishing another $1.0 \mu \mathrm{L}$ crystallization solution onto the crystal as exhibited in Fig. 5. More detailed crystal morphologies and the corresponding CFDCs were displayed in Supplementary Fig. 13. Concretely, the size of CFDC was enlarged by injecting more crystallization solution on much larger sessile platform (also constructed via 3D printing), whose size was correspondingly enlarged to match the enlarged CFDC $\left(120^{\circ}\right)$. The side views of resultant CFDCs and the corresponding crystal morphologies after being completely dried were probed by contact angle goniometry and SEM, respectively (Fig. 5a, b). To evaluate such scale-up and the effect of gravity on the flexible gas-liquid interface, an error of CFDC shape was defined as the ratio of aspect ratio for actual and ideal CFDC, as exhibited in Supplementary Fig. 14. It was worth noting that the aspect ratio of the actual CFDC $(1 \mu \mathrm{L})$ was 0.766 while the ideal CFDC $\left(120^{\circ}\right)$ was 0.750 , which exhibited an error of $2.16 \%$ as displayed in Supplementary Table 2. When the volume of the enlarged CFDC was below $16.0 \mu \mathrm{L}$ (16 times in volume), the error of aspect ratio was lesser than $8.0 \%$ although suffering the gravity. This should be attributed to the effect of surface tension, which overcame the gravitational effect onto the flexible gas-liquid interface of CFDC (Fig. 5a). Thus, the desired synergized micro-flows were maintained inside CFDC, which conveyed the solutes and micro-crystals towards sessile platform center through the center shaft region. And then the standard cubic crystal was scaled up at the center of sessile platform (Fig. 5a, b). While, when the volume of CFDC was above $20.0 \mu \mathrm{L}$, this scale-up was failed with the excessive error of aspect ratio above $10.0 \%$ due to the intensified gravitational effect. Thereby, the perfect synergized micro-flows were destroyed, resulting in the disappearance of cubic crystal at the sessile platform center (Fig. 5b).

In addition, benefiting from the flexibility of droplet, new CFDC can be consecutively constructed on the initial CFDC. Thus, the stage-up (secondary to forth feeding) of cubic crystal was realized for diverse CFDCs by replenishing another $1.0 \mu \mathrm{L}$ crystallization solution onto the original crystal (Fig. 5c, d). This not only caused the dissolution of original coffee-ring or cubic crystals, but also accomplished the regrowth of new crystal during the droplet crystallization (as manifested in Supplementary Videos 2, 3). Compared with the original crystals, the crystal morphology was normalized to a regular cubic crystal with much larger crystal size. As shown in Fig. 5c, the classical coffee-ring crystal was obtained by 
evaporating $0.4 \mu \mathrm{L} \mathrm{NaCl}$ solution, which constructed a small CFDC $\left(90^{\circ}\right)$ on sessile platform. In this case, the capillary flow dominated the formation of synergized micro-flows inside CFDC and thus conveyed the solutes and/or micro-crystals towards contact line region, acquiring a coffee-ring crystal along the contact line. After replenishing another $1.0 \mu \mathrm{L}$ solution, a new CFDC $\left(120^{\circ}\right)$ was constructed on the sessile platform, where the coffee-ring crystal was dissolved and reassembled a new large cubic crystal (from $1.4 \mu \mathrm{L}$ solution) at sessile platform center by optimizing the match of capillary flow and Marangoni flow (Fig. 5c). Besides, when the new CFDC (about $120^{\circ}$ ) was established by replenishing another $1.0 \mu \mathrm{L}$ solution onto the residual droplet with nascent cubic crystal, the original cubic crystal was dissolved, and then a much larger cubic crystal was attained at the center of sessile platform from $2.0 \mu \mathrm{L}$ solution (Fig. $5 \mathrm{~d})$. These results collectively indicated that the perfect synergy of capillary flow and Marangoni flow can be fully maintained during the scale-up and stage-up operation, until a certain degree of scale-up (16 times) and stage-up (2 to 4 times). Thus, the CFDC constructed on the developed sessile platform had great potential, feasibility, and adjustability in engineering the crystallization process.

Considering the effect of gravity on the droplet, hanging droplet method was regarded as a common strategy for micro-droplet crystallization (especially for biomacromolecule crystal preparation) ${ }^{49-52}$. In this study, based on the virtues of sessile platform and CFDC, we inverted the sitting CFDC $\left(120^{\circ}\right)$ to construct a hanging CFDC on the reversed sessile platform (Fig. 6). And then a potential sheet crystal was appeared and developed along the gas-liquid interface. Ultimately, the sheet crystal was fully developed and restricted on the sessile platform center by surface tension as manifested in Fig. 6a and Supplementary Video 4. By contrast, a cubic crystal could be obtained after the growth of initial crystal surrounded by crystallization solution. To analyze the crystallization mechanism of sitting and hanging CFDCs $\left(120^{\circ}\right)$, CFD simulations were applied to probe the temperature, concentration, and velocity distributions, thereby evaluating the synergized micro-flows mechanism during crystallization process (Supplementary Fig. 15). Influenced by the reversed gravity direction, the temperature and concentration distributions inside the hanging CFDC were dramatically changed when it was compared with that of sitting CFDC (Fig. 6b, c). Particularly, the orientation of the center shaft region was reversed towards the gas-liquid interface. Besides, a higher value of corner $(296.2 \mathrm{~K})$ in temperature distribution and lower peak $(22.0 \%)$ in concentration distribution along the axis of hanging CFDC were observed while those of sitting CFDC were $295.6 \mathrm{~K}$ and $22.6 \%$, respectively (Fig. 6e, f), which signified the declined driving force in the hanging CFDC. This was verified by the reduced peak value of velocity distribution from 0.072 to $0.054 \mathrm{~mm} / \mathrm{s}$ compared with the sitting CFDC (Fig. $6 \mathrm{~g}$ ). Meanwhile, the shifts of corner and peak positions in temperature and concentration distributions from 0.66 to $0.76 \mathrm{~mm}$ induced the similar shift of peak position (velocity distribution) towards gas-liquid interface of CFDCs. These results further testified the migration of center shaft region towards the gas-liquid interface, which was evidenced by the velocity vector as exhibited in Fig. $6 \mathrm{~d}$. This shift conveyed solutes and/or micro-crystals towards the gas-liquid interface (hanging CFDC), rather than the sessile platform center (sitting CFDC). Therefore, for the crystal with higher density than the solution, the sitting CFDC may possess much larger potential on crystal nucleation and growth in the investigated system, paving the way for the preparation of cubic crystal at the sessile platform center. In addition, the developed 3D-printed matrix-type and regular platform had 
illustrated multiple functions of revelation the undergoing crystallization mechanism and parallel preparation the target crystal in diverse CFDCs, etc.

\section{Discussion}

In summary, high precision 3D printing technology were induced to prepare a micro device with many designed sessile platforms, which exhibited excellent sessile function of droplet. And then, benefiting from the flexible gas-liquid interface, the nanoliter CFDCs with different morphologies were confined on the sessile platform by precisely tuning the droplet volume. Based on the regulated CFDC shapes, we controlled the mass-heat transfer and the assembly of the crystal inside CFDCs, further revealing synergized micro-flows mechanism by integrating the capillary flow and Marangoni flow via CFD simulations. Instructed by this mechanism, a standard cubic crystal was obtained at the sessile platform center through a perfect match of capillary flow and Marangoni flow in a moderate CFDC $\left(120^{\circ}\right)$. Moreover, the scale-up of the platform or supplementary feeding (secondary to forth feeding) can facilely realize the preparation of larger standard cubic crystal at sessile platform center, even enlarging the crystal size to 16 times, which exhibited great potential on crystal morphology regulation, crystallization mechanism revelation, and high-quality crystal harvest. Compared with the stationary interface in tank and tube crystallizers, the flexible interface originated from the integration of liquid surface tension and sessile platform subtly synergized the space and intensity of micro-flows. This endowed CFDC with facilely modulation of crystallization process than that of crystallizer with stationary interface, holding great potential to the fast screen of fine crystallization condition and the investigation of nanoparticle behavior in droplet.

\section{Methods}

Materials: Hydrophilic photoresist resin ( 90\% poly-acrylics acid, PAA, MW 1500 to $2000 \mathrm{~g} \mathrm{~mol}^{-1}$ ) was supplied by Guangzhou 4.0 Industry Design Co., Ltd. Sodium chloride ( $\mathrm{NaCl}, \mathrm{AR})$ and ethanol (AR) were purchased from Tianjin Tianli Chemical Reagent Co., Ltd. Ammonium hydroxide ( $25 \%$ solution in water) was obtained from Aladdin Chemical Co., Ltd. Nanoparticle with radius of $200 \mathrm{~nm}$ was provided by PuriMag Biotechnology Co., Ltd. All chemicals were utilized as received, and deionized water was used throughout the experiments.

Synthesis of sessile platform: High precision digital light process 3D printer was used to prepare the micro platform (radius of $600 \mu \mathrm{m}$ ). Concretely, the model date was analyzed by 3D printer layer by layer after converting the digital model. During the fabrication, the precision of 3D printer was set as $12.5 \mu \mathrm{m}$ in $\mathrm{z}$ axis and $50 \mu \mathrm{m}$ in $\mathrm{x}$ and $\mathrm{y}$ axes. Meanwhile, the temperature was maintained at about $25^{\circ} \mathrm{C}$ while the humidity is around $40 \%$. Subsequently, the micro platform was cleaned in ethanol with ultrasonic for 5 min to remove the residual resin. Then, a high-power UV lamp (1000 W) was utilized to ensure the completely solidification of micro platform. Additionally, the micro platform was immersed in $25 \%$ ammonium hydroxide solution for $48 \mathrm{~h}$ to neutralize the residual carboxyl groups on micro platform surface. After enough ion exchange with $\mathrm{NaCl}(1 \mathrm{M})$ solution and water cleaning, a neutral micro platform 
was obtained, which avoided the electrostatic interaction between solutes and platform surface during the droplet crystallization. Ultimately, a sessile platform with advantages of neutral, hydrophilic, and flat was prepared after a fully sterilization $(24 \mathrm{~h})$ in ethanol. Following this preparation, much larger sessile platforms with increased radius of $0.76,0.95,1.09,1.37,1.51,1.63$, and $1.82 \mathrm{~mm}$ were also designed and fabricated, which maintained the optimal droplet contact angles $\left(120^{\circ}\right)$ while the droplet volume was correspondingly increased from 1.0 to $2.0,4.0,6.0,12.0,16.0,20.0$, and $28.0 \mu \mathrm{L}$.

Preparation of sample solution and regulation of operation condition: To guarantee repeatability of droplet crystallization process, $\mathrm{NaCl}$ was chosen to analyze the synergized micro-flows mechanism due to the insensitive solubility for temperature and humidity. The targeted $\mathrm{NaCl}$ solution with $20 \%$ saturability was obtained by diluting $4.0 \mathrm{~mL}$ deionized water into $1.0 \mathrm{~mL}$ saturated $\mathrm{NaCl}$ solution. In addition, the operation condition for all droplet crystallization was restricted as temperature ranged $25 \pm 3$ ${ }^{\circ} \mathrm{C}$ and humidity ranged $40 \pm 3 \%$ in the crystallization system.

Construction of CFDCs: In this work, the CFDCs with different morphologies were achieved by precisely tuning the droplet volume through a droplet dripping system. Confined by the hydrophilic sessile platform, the CFDCs with contact angles from $51^{\circ}$ to $140^{\circ}$ were facilely obtained although the inherent contact angle of sessile platform was $77^{\circ}$. Besides, the hanging CFDC $\left(120^{\circ}\right)$ was acquired by reversing the sitting CFDC $\left(120^{\circ}\right)$.

Characterization of sessile platform, CFDCs and crystal morphologies: The contact angle of CFDC was estimated by Drop Shape Analyzer (DSA30M, Germay Kruss). The morphology of sessile platform was characterized by scanning electron microscopy (SEM, Auriga FIB SEM, Zeiss, Germany) and atomic force microscopy (AFM, Bruker Dimension ICON). The chemical composition of sessile platform surface was analyzed by zeta potential analyzer, X-ray photoelectron spectroscopy (XPS, AXIS Supra, Kratos, UK) and energy-dispersive spectroscopy (EDS) on SEM instrument. Crystal morphology was detected by SEM (Hitachi TM3000). The side views of CFDC and crystal were observed and captured by a stereo microscope (SMZ25, Nikon, Japan). The temperature distribution along the gas-liquid interface was detected by an infrared thermal imager (Fluke TiX580). Meanwhile, the evolutions of CFDC and microflows inside droplet were recorded by an on-line camera on Drop Shape Analyzer. Both of them are connected with a computer.

CFD simulations: In this paper, the synergized micro-flows inside CFDCs were simulated by CFD simulation, in which the $\mathrm{NaCl}$ solution (20\% saturability) was selected due to the insensitive solubility for temperature and humidity. To achieve such $\mathrm{NaCl}$ solution, the ratio of saturated solution and water was set as 1:4 inside all CFDCs. After an enough development (31 s), a CFDC with stable temperature, concentration, and velocity distributions can be obtained. Particularly, the temperature and concentration fields and internal fluidity of sodium chloride droplets on microscale sessile platform are investigated. The conservation equations including continuity, momentum, and energy equations are incorporated and solved numerically in accordance with the conditions of experiment. The governing equations could be written as the following form. 
$\frac{\partial \rho}{\partial t}+\nabla \cdot(\rho \vec{V})=0$

where $\rho$ is the fluid density and $\vec{V}$ is the fluid velocity.

$\frac{D \vec{V}}{D t}=\overrightarrow{f_{b}}-\frac{1}{\rho} \nabla p+\frac{\mu}{\rho} \nabla^{2} \vec{V}+\frac{1}{3} \frac{\mu}{\rho} \nabla(\nabla \cdot \vec{V})$

where $f_{b}$ is the volume force term, $\mathrm{p}$ is the pressure, $\frac{\mu}{\rho} \nabla^{2} \vec{V}+\frac{1}{3} \frac{\mu}{\rho} \nabla(\nabla \cdot \vec{V})$ is viscous force term,

$\mu$ is the dynamic viscosity of the liquid. The fluid flow in droplet is regarded as laminar flow of incompressible Newtonian fluid. Thus, the $\frac{1}{3} \frac{\mu}{\rho} \nabla(\nabla \cdot \vec{V})$ can be ignored.

$$
\rho \frac{D}{D t}\left[u+\frac{V^{2}}{2}\right]=\rho \overrightarrow{f_{b}} \cdot \vec{V}+\nabla \cdot\left(\vec{V} \cdot \tau_{i j}\right)+\nabla(\lambda \nabla T)+\rho \dot{q}
$$

In order to make the simulation results more consistent with the experimental data, 3D modeling of sodium chloride droplets was carried out by software, and the fluid domain of droplets was created. Then, ICEM software is used to mesh the computational domain, in which tetrahedral mesh is used. In the numerical approach, finer grid points were used for the boundary area and the grid independence tests were carried out to secure the grid independent solutions. After accomplishing the grid independence tests, the mesh size comprising of $1.11 \mathrm{M}$ cells is selected and adapted in the simulations.

Fluent solver is used to solve the mesh. Based on the mixture model, two phases are considered, with pure water solution as the main phase and solute phase as the secondary phase. Consider gravitational acceleration and the loss of mass, momentum and heat due to evaporation. The SIMPLE algorithm is used to solve the problem iteratively. In the Spatial Discretization panel, the Gradient selects Least Squares Cell Based, the Pressure selects Second Order, the Momentum selects Second Order Upwind, the Energy selects Second Order Upwind. The implicit scheme with a backward difference approximation was used and unconditionally stable solutions are ensured. The selection of time step was critical to ensure the accuracy of the scheme; in which case, it was set in the order of $0.01 \mathrm{~s}$. The residuals of flow parameters are set as 0.001 .

\section{Declarations}

\section{Data availability}

All data are available in the main text and the supplementary materials.

\section{Acknowledgements}

We acknowledge the financial contribution from Science Fund for Creative Research Groups of the National Natural Science Foundation of China (22021005), National Natural Science Foundation of China (Grant No. 21978037), Fundamental Research Funds for the Central Universities (DUT19TD33), and 
National Key Research and Development Program of China (Grant No. 2019YFE0119200), Support Plan of Innovative Talents of Liaoning Province (XLYC1901005, XLYC1907149), Dalian Innovative Science and Technology Fund (2020JJ26SN064).

\section{Author contributions}

X. J. and G. H. conceived and designed the project. Z. Y. performed the experiments, developed the model, and wrote the paper. X. J. revised the paper. Z. L. discussed the CFD simulation results. Y. W. discussed the designation of experiments. All authors discussed the results.

\section{Competing interests}

The authors declare no competing interests

\section{Additional information}

Supplementary information is available for this paper.

Correspondence and requests for materials should be addressed to X. J. and G. H.

\section{References}

1. Cai, Q. W. et al. Controllable Fabrication of Functional Microhelices with Droplet Microfluidics. ACS Appl. Mater. Interfaces 11, 46241-46250 (2019).

2. Sun, H. H. et al. Evaporation of saline colloidal droplet and deposition pattern. Chinese Phys. B 29, (2020).

3. Li, Y., Salvator, V., Wijshoff, H., Versluis, M. \& Lohse, D. Evaporation-Induced Crystallization of Surfactants in Sessile Multicomponent Droplets. Langmuir 36, 7545-7552 (2020).

4. Jiang, X. et al. Interfacial microdroplet evaporative crystallization on 3D printed regular matrix platform. AlChE J. 66, (2020).

5. Wang, S. et al. Directed motion of an impinging water droplet - Seesaw effect. J. Mater. Chem. A 8, 7889-7896 (2020).

6. Han, M. et al. Tailored 3D printed micro-crystallization chip for versatile and high-efficiency droplet evaporative crystallization. Lab Chip 19, 767-777 (2019).

7. Koishi, A. et al. Surface Wetting Controls Calcium Carbonate Crystallization Kinetics. Chem. Mater. 31, 3340-3348 (2019).

8. Du, L., Wang, Y. \& Luo, G. In situ preparation of hydrophobic CaCO3 nanoparticles in a gas-liquid microdispersion process. Particuology 11, 421-427 (2013).

9. Du, L., Wang, Y., Wang, K. \& Luo, G. Preparation of calcium benzene sulfonate detergents by a microdispersion process. Ind. Eng. Chem. Res. 52, 10699-10706 (2013). 
10. Hu, Y. et al. The amino-terminal structure of human fragile $X$ mental retardation protein obtained using precipitant-immobilized imprinted polymers. Nat. Commun. 6, 1-11 (2015).

11. Basu, N. \& Mukherjee, R. Morphology modulation in evaporative drying mediated crystallization of sodium chloride solution droplet with surfactant. Soft Matter 14, 7883-7893 (2018).

12. Kwieciński, W. et al. Evaporation of Dilute Sodium Dodecyl Sulfate Droplets on a Hydrophobic Substrate. Langmuir 35, 10453-10460 (2019).

13. Ozturk, T. \& Erbil, H. Y. Evaporation of water-ethanol binary sessile drop on fluoropolymer surfaces: Influence of relative humidity. Colloids Surfaces A Physicochem. Eng. Asp. 553, 327-336 (2018).

14. Lum, A. et al. Role of Steam as a Medium for Droplet Crystallization. Ind. Eng. Chem. Res. 58, 85178524 (2019).

15. Basu, N. \& Mukherjee, R. Evaporative Drying of Sodium Chloride Solution Droplet on a Thermally Controlled Substrate. J. Phys. Chem. B 124, 1266-1274 (2020).

16. Cheng, M. et al. Parallel and Precise Macroscopic Supramolecular Assembly through Prolonged Marangoni Motion. Angew. Chemie Int. Ed. 130, 14302-14306 (2018).

17. Lee, H. H., Fu, S. C., Tso, C. Y. \& Chao, C. Y. H. Study of residue patterns of aqueous nanofluid droplets with different particle sizes and concentrations on different substrates. Int. J. Heat Mass Transf. 105, 230-236 (2017).

18. Liang, M. et al. Shape evolution and thermal stability of lysozyme crystals: Effect of $\mathrm{pH}$ and temperature. Bioprocess Biosyst. Eng. 36, 91-99 (2013).

19. Zhao, J. et al. Modulating Self-Assembly of DNA Crystals with Rationally Designed Agents. Angew. Chemie Int. Ed. 130, 16767-16770 (2018).

20. Li, F. \& Lakerveld, R. Influence of Alternating Electric Fields on Protein Crystallization in Microfluidic Devices with Patterned Electrodes in a Parallel-Plate Configuration. Cryst. Growth Des. 17, 30623070 (2017).

21. Misyura, S. Y. Different modes of heat transfer and crystallization in a drop of $\mathrm{NaCl}$ solution: The influence of key factors on the crystallization rate and the heat transfer coefficient. Int. J. Therm. Sci. 159, 106602 (2021).

22. Misyura, S. Y. Heat transfer and convection of evaporating sessile droplets in transition from superhydrophilic to superhydrophobic structured wall: Optimization of functional properties. Int. Commun. Heat Mass Transf. 112, 104474 (2020).

23. Van der Weijden, A., Winkens, M., Schoenmakers, S. M. C., Huck, W. T. S. \& Korevaar, P. A. Autonomous mesoscale positioning emerging from myelin filament self-organization and Marangoni flows. Nat. Commun. 11, 1-10 (2020).

24. Tiribocchi, A. et al. The vortex-driven dynamics of droplets within droplets. Nat. Commun. 12, (2021).

25. Almohammadi, H., Bagnani, M. \& Mezzenga, R. Flow-induced order-order transitions in amyloid fibril liquid crystalline tactoids. Nat. Commun. 11, 1-9 (2020).

26. Chen, Y. et al. Morphology selection kinetics of crystallization in a sphere. Nat. Phys. 17, (2021). 
27. Yunker, P. J., Still, T., Lohr, M. A. \& Yodh, A. G. Suppression of the coffee-ring effect by shapedependent capillary interactions. Nature 476, 308-311 (2011).

28. Han, W. \& Lin, Z. Learning from 'coffee rings': Ordered structures enabled by controlled evaporative self-assembly. Angew. Chemie Int. Ed. 51, 1534-1546 (2012).

29. Hu, H. \& Larson, R. G. Marangoni effect reverses coffee-ring depositions. J. Phys. Chem. B 110, 7090-7094 (2006).

30. Xu, X. \& Luo, J. Marangoni flow in an evaporating water droplet. Appl. Phys. Lett. 91, (2007).

31. Buffone, C., Sefiane, K. \& Christy, J. R. E. Experimental investigation of self-induced thermocapillary convection for an evaporating meniscus in capillary tubes using micro-particle image velocimetry. Phys. Fluids 17, 1-18 (2005).

32. Buffone, C. \& Sefiane, K. Investigation of thermocapillary convective patterns and their role in the enhancement of evaporation from pores. Int. J. Multiph. Flow 30, 1071-1091 (2004).

33. Hu, H. \& Larson, R. G. Analysis of the effects of marangoni stresses on the microflow in an evaporating sessile droplet. Langmuir 21, 3972-3980 (2005).

34. Misyura, S. Y. The influence of convection on heat transfer in a water layer on a heated structured wall. Int. Commun. Heat Mass Transf. 102, 14-21 (2019).

35. Yu, Y., Sun, L., Huang, X. \& Zhou, J. Evaporation of ethanol / water mixture droplets on a pillar-like PDMS surface. Colloids Surfaces A 574, 215-220 (2019).

36. Hu, H. \& Larson, R. G. Analysis of the microfluid flow in an evaporating sessile droplet. Langmuir 21, 3963-3971 (2005).

37. Deegan, R. D. et al. Capillary flow as the cause of ring stains from dried liquid drops. Nature $\mathbf{3 8 9}$, 827-829 (1997).

38. Davidson, Z. S. et al. Deposition and drying dynamics of liquid crystal droplets. Nat. Commun. 8, 1-7 (2017).

39. Wray, A. W. et al. geometrically-controlled sessile drop evaporation. 1-9 (2017) doi:10.1038/ncomms14783.

40. Mampallil, D. \& Eral, H. B. A review on suppression and utilization of the coffee-ring effect. Adv. Colloid Interface Sci. 252, 38-54 (2018).

41. Zhang, T. H. \& Liu, X. Y. Nucleation: What happens at the initial stage? Angew. Chemie Int. Ed. 48, 1308-1312 (2009).

42. Zhang, Z. et al. Controlled inkjetting of a conductive pattern of silver nanoparticles based on the coffee-ring effect. Adv. Mater. 25, 6714-6718 (2013).

43. Deegan, R. D. et al. Contact line deposits in an evaporating drop. Phys. Rev. E-Stat. Physics, Plasmas, Fluids, Relat. Interdiscip. Top. 62, 756-765 (2000).

44. Still, T., Yunker, P. J. \& Yodh, A. G. Surfactant-induced Marangoni eddies alter the coffee-rings of evaporating colloidal drops. Langmuir 28, 4984-4988 (2012). 
45. Sempels, W., De Dier, R., Mizuno, H., Hofkens, J. \& Vermant, J. Auto-production of biosurfactants reverses the coffee ring effect in a bacterial system. Nat. Commun. 4, 1-8 (2013).

46. Mayarani, M., Basavaraj, M. G. \& Satapathy, D. K. Viscoelastic Particle-Laden Interface Inhibits Coffee-Ring Formation. Langmuir 34, 14294-14301 (2018).

47. Talbot, E. L. et al. Printing small dots from large drops. ACS Appl. Mater. Interfaces 7, 3782-3790 (2015).

48. Anyfantakis, M., Baigl, D. \& Binks, B. P. Evaporation of Drops Containing Silica Nanoparticles of Varying Hydrophobicities: Exploiting Particle-Particle Interactions for Additive-Free Tunable Deposit Morphology. Langmuir 33, 5025-5036 (2017).

49. Guo, Y. Z., Yin, D. C., Lu, Q. Q., Wang, X. K. \& Liu, J. Enhancement of nucleation during hanging drop protein crystallization using HF Treatment of cover glasses. Cryst. Res. Technol. 45, 158-166 (2010).

50. Hou, H. et al. A systematic comparison of sitting and hanging-drop crystallization using traditional and cross-di ff usion microbatch crystallization plates. J. Cryst. Growth 521, 1-8 (2019).

51. Meldrum, F. C. \& O’Shaughnessy, C. Crystallization in Confinement. Adv. Mater. 32, (2020).

52. Watanabe, T., Takakusagi, T., Ueno, I., Kawamura, H. \& Nishino, K. International Journal of Heat and Mass Transfer Terrestrial and microgravity experiments on onset of oscillatory thermocapillarydriven convection in hanging droplets. Int. J. Heat Mass Transf. 123, 945-956 (2018).

\section{Figures}

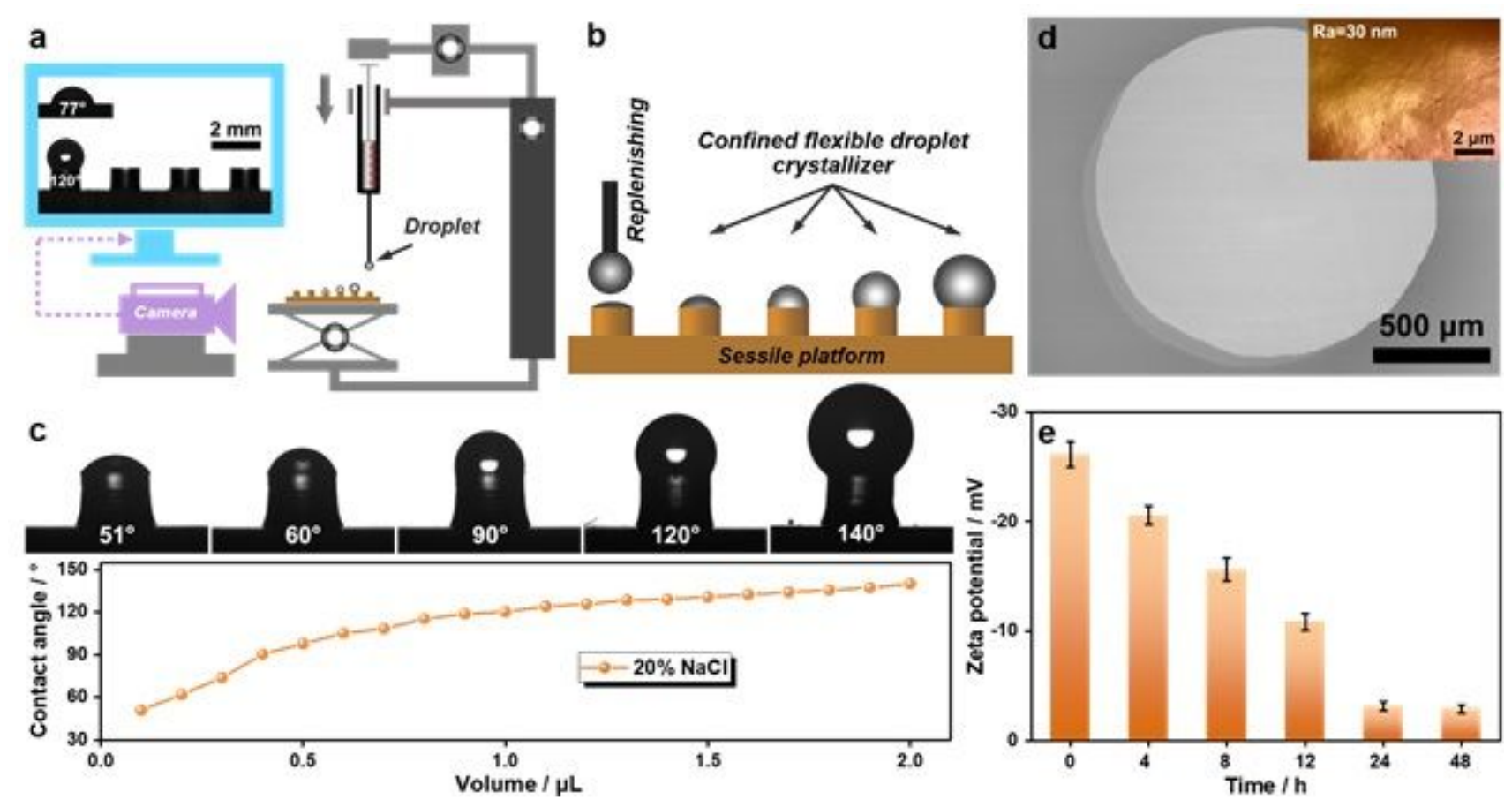

Figure 1 
Construction of CFDCs on 3D printed sessile platform. a Schematic diagram of the nanoliter droplet dripping system and on-line monitor experiment setup; Inset displays that the side view and inherent contact angle of the sessile platform. b Illustration of diverse CFDCs and the replenishment of crystallization solution. c The SEM and AFM images of sessile platform surface. $d$ Side view and contact angle against the volume for CFDC. e Zeta potential against the time of neutralization for sessile platform surfaces at $\mathrm{pH}=7$.

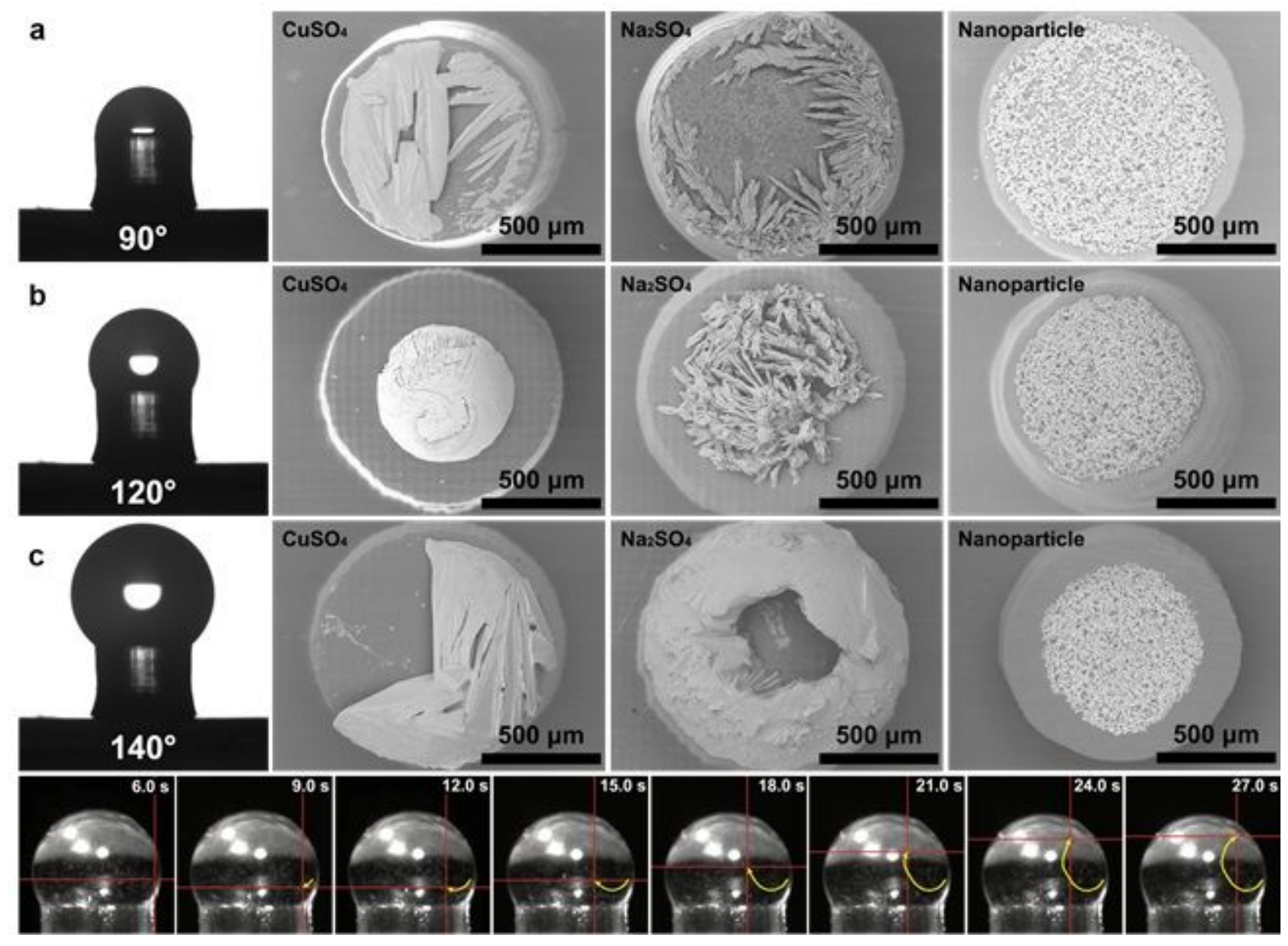

Figure 2

Proofs for the enhanced Marangoni flow intensity with increased initial contact angles of CFDCs. The side views and deposition morphologies of $\mathrm{CuSO} 4, \mathrm{Na} 2 \mathrm{SO} 4$, and nanoparticles for the CFDCs with contact angle of $90^{\circ}(\mathrm{a}), 120^{\circ}(\mathrm{b})$, and $140^{\circ}$ (c). $d$ Trajectory of one nanoparticle conveyed by synergized micro-flows during the evaporation. 

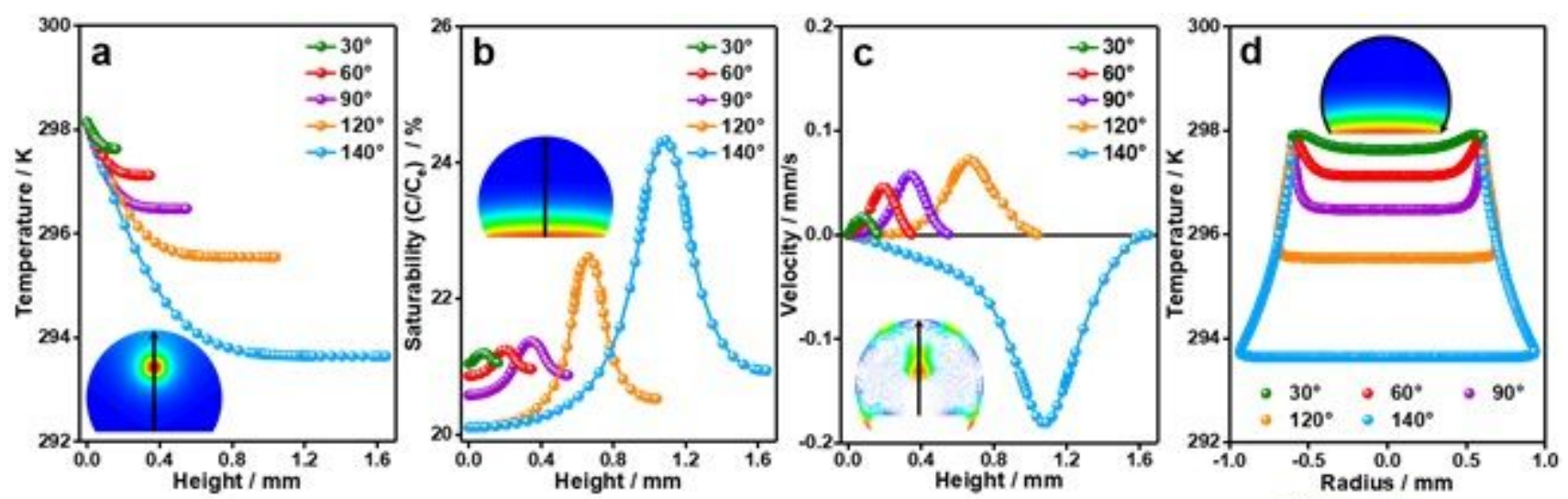

e

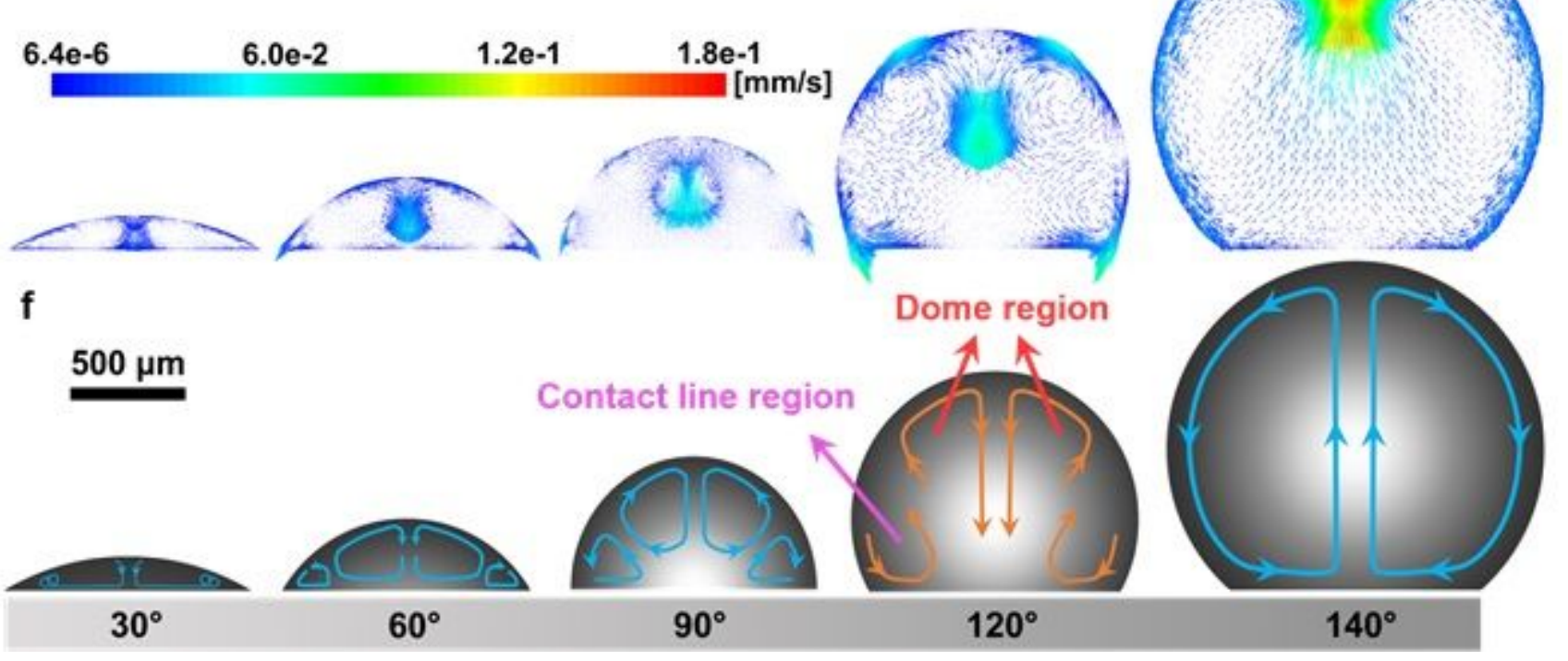

Figure 3

Proofs for the synergy of capillary flow and Marangoni flow. The temperature (a), concentration (b), and velocity (c) distributions along the axes and the temperature distributions along the gas-liquid interface (d) of CFDCs with initial contact angles of $30^{\circ}, 60^{\circ}, 90^{\circ}, 120^{\circ}$, and $140^{\circ}$. Noting the $\mathrm{C}$ and $\mathrm{Ce}(\mathrm{b})$ are defined as the concentration of crystallization solution and saturated solution, respectively. The simulation (e) and illustration (f) results of the synergized micro-flows inside CFDCs with initial contact angles of $30^{\circ}, 60^{\circ}, 90^{\circ}, 120^{\circ}$, and $140^{\circ}$. 


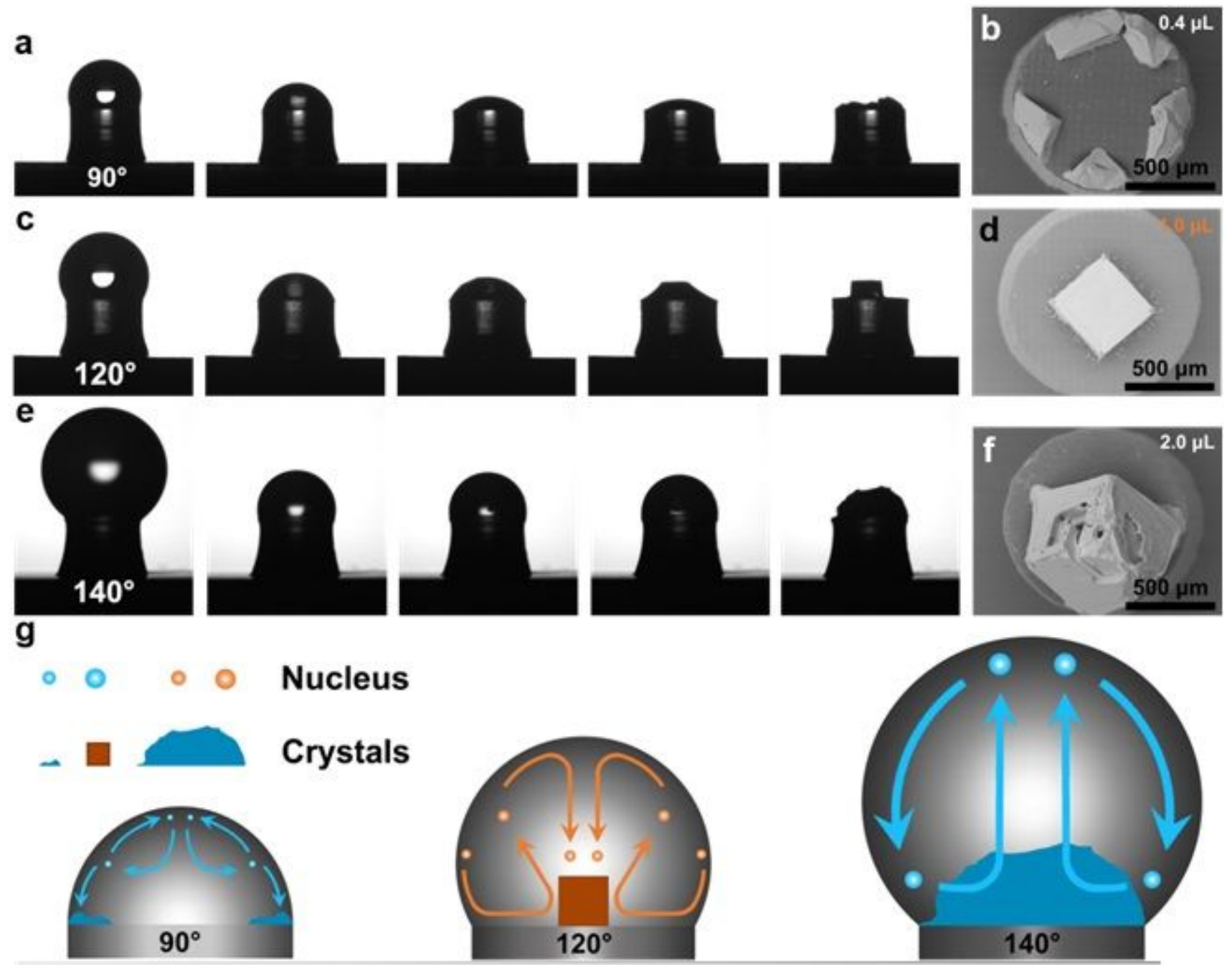

Figure 4

The precise preparation of standard cubic crystal at sessile platform center. The side views of CFDCs with initial contact angles of $90^{\circ}$ (a), $120^{\circ}$ (c), and $140^{\circ}$ (e) during the evaporation crystallization. The SEM images of crystal morphologies with initial contact angles of $90^{\circ}$ (b), $120^{\circ}$ (d), and $140^{\circ}$ (f) after evaporation crystallization. g Illustrations of micro-crystal transfer mechanism induced by the synergized micro-flows inside CFDCs with initial contact angles of $90^{\circ}, 120^{\circ}$, and $140^{\circ}$. 


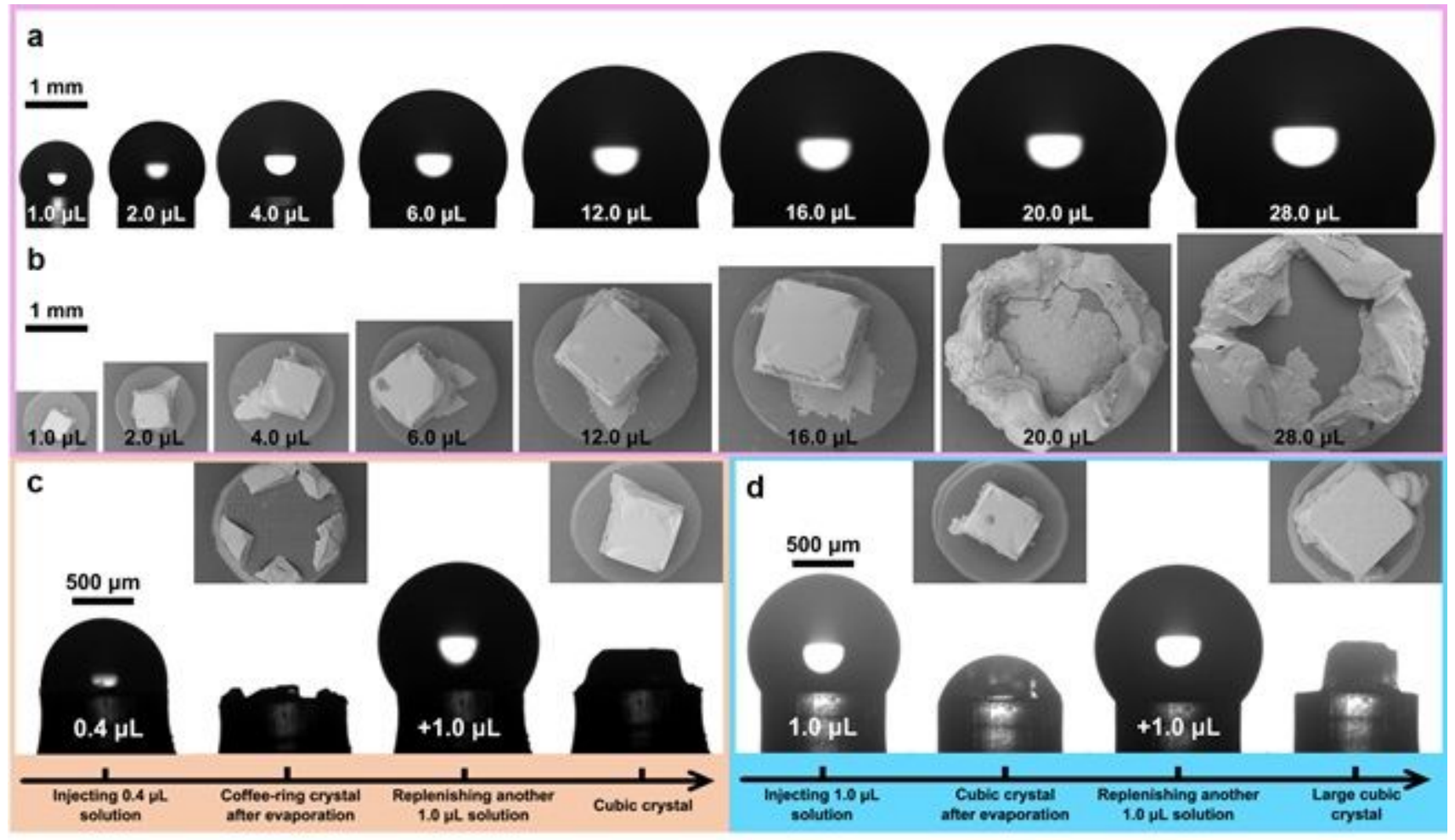

Figure 5

Proofs for the scale-up and stage-up of standard cubic crystal via CFDC. The side views of CFDCs (a) and top views of crystal morphologies (b) for the scale-up of cubic crystal. The stage-up of cubic crystal from coffee-ring crystal (c) and cubic crystal (d) by injecting another $1.0 \mu \mathrm{L}$ solution. 


\section{a}
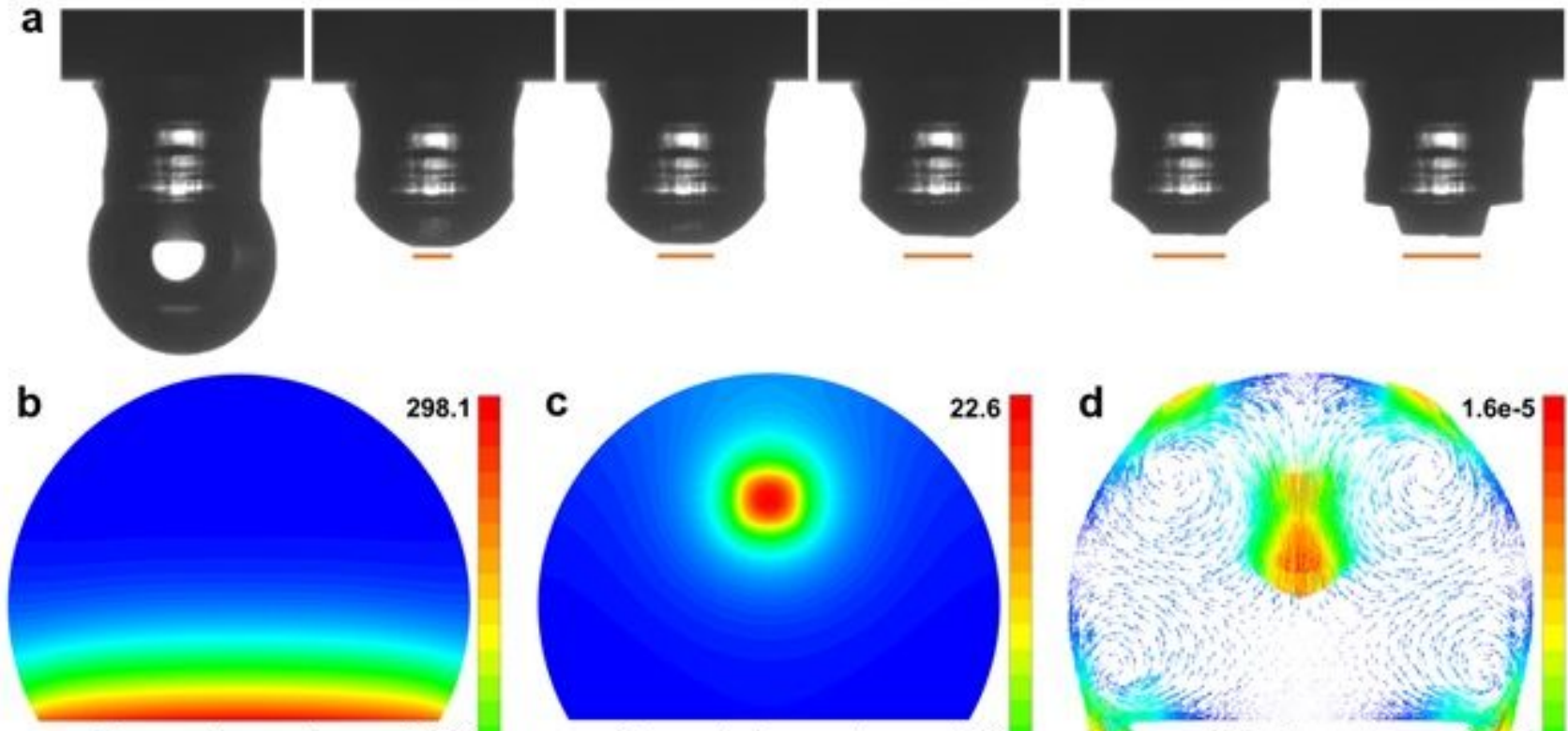

Temperature contour

296.8

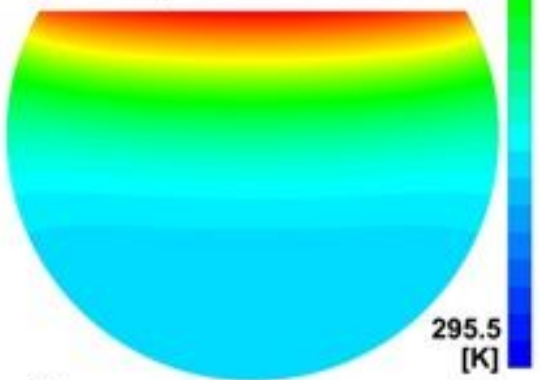

Concentration contour

Velocity vector $\quad 3.7 \mathrm{e}-2$
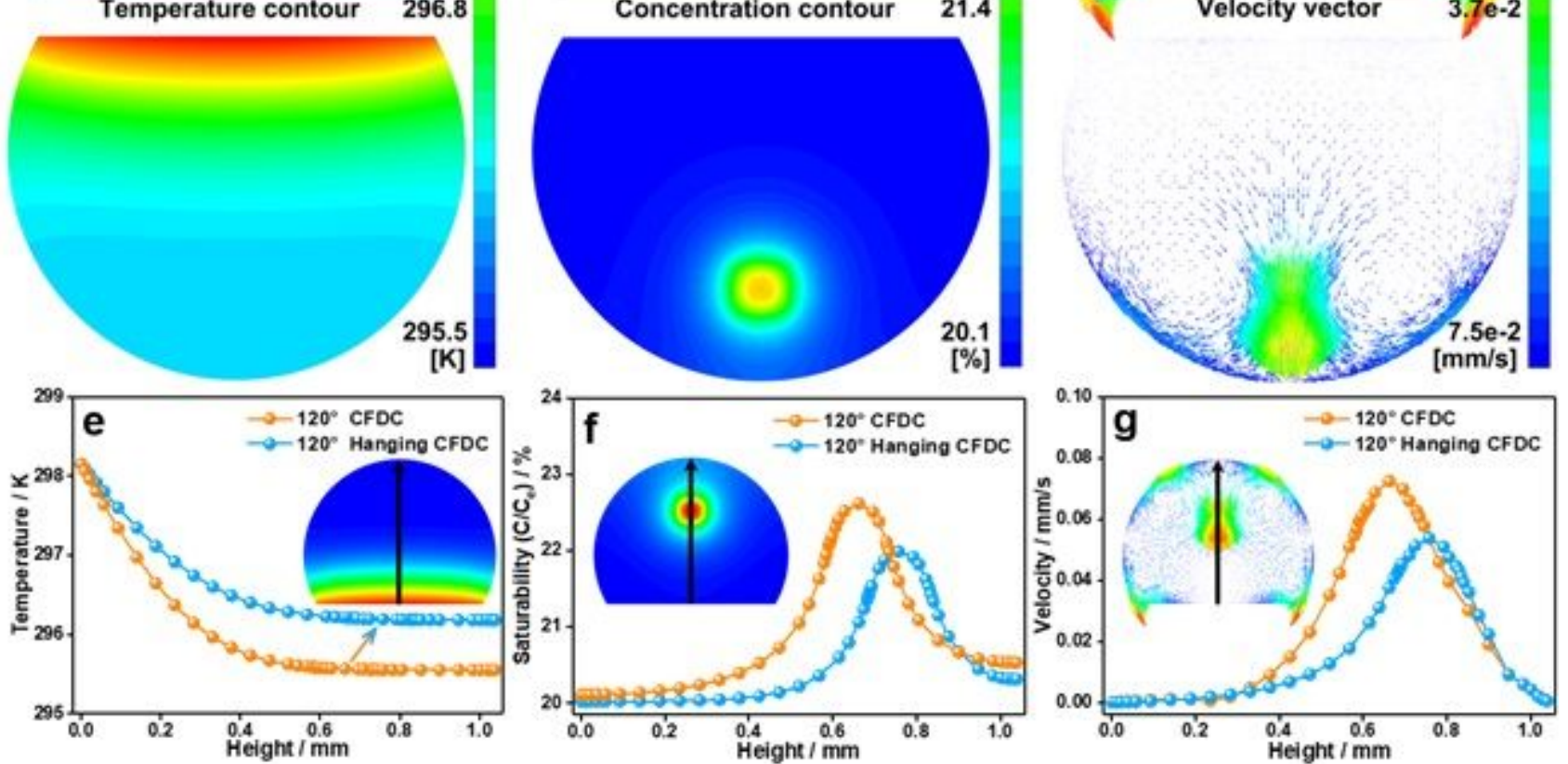

Figure 6

Proofs for the cubic crystal preparation of sitting and hanging CFDCs. a Crystallization process of hanging CFDC with the initial contact angle of $120^{\circ}$. The temperature contours (b), concentration contours (c), and velocity vectors (d) projections on the cross-section plane of sitting (top) and hanging (bottom) CFDCs with initial contact angle of $120^{\circ}$. The temperature (e), concentration ( $\mathrm{f}$ ), and velocity (g) distributions of sitting and hanging $\mathrm{CFDCs}\left(120^{\circ}\right)$ along the axis.

\section{Supplementary Files}

This is a list of supplementary files associated with this preprint. Click to download.

- Video190XX.mp4 
- Video2.mp4

- Video4.mp4

- Video3.mp4

- Supplementary20210511.docx 